\title{
Ética del Consumo en la Gestión de la Seguridad Alimentaria en el Cantón Santo Domingo de los Colorados, Ecuador
}

\author{
Milton C. Barragán ${ }^{(1)}$ y Dante Ayaviri(2) \\ (1) Facultad de Recursos Naturales, Instituto de Posgrado y Educación Continua, Escuela Superior \\ Politécnica de Chimborazo, Riobamba-Ecuador. (e-mail: mcbarragan@pronaca.com) \\ (2) Facultad de Ciencias Políticas y Administrativas, Universidad Nacional de Chimborazo, Riobamba- \\ Ecuador. (e-mail: vdayaviri@gmail.com)
}

Recibido Dic. 1, 2017; Aceptado Feb. 9, 2018; Versión final Abr. 9, 2018, Publicado Oct. 2018

\section{Resumen}

Se analiza y determina cómo la ética del consumo de la población urbana contribuye o no en la gestión de la seguridad alimentaria de Santo Domingo de los Colorados, Ecuador. Para obtener los datos se empleó técnicas cualitativas y cuantitativas como entrevistas y encuestas aplicando un muestreo aleatorio estratificado en función del nivel de ingresos. Los resultados muestran que el $51,3 \%$ de la población no es consciente de los impactos ambientales y sociales que se generan por los hábitos de consumo actuales. Los problemas sociales y ambientales relacionados con el consumo son la contaminación por basura doméstica, contaminación de fuentes de agua, uso excesivo de agroquímicos, desperdicio de alimentos e incremento de obesidad. La población está dispuesta a pagar un precio más alto por productos con responsabilidad social empresarial, reducir el desperdicio de alimentos, realizar reciclaje y reducir el consumo de alimentos para contribuir a la seguridad alimentaria. En conclusión, la población no realiza actualmente un consumo ético, provocando varios problemas sociales y ambientales que impiden el logro de la seguridad alimentaria de todos los habitantes.

\section{Ethics of Consumption in Food Security Management in the Canton of Santo Domingo de los Colorados, Ecuador}

\begin{abstract}
The objective of the present investigation was to determine how the ethics of consumption of the urban population contributes or not in the management of the food safety of Santo Domingo de los Colorados, Ecuador. To obtain the data, qualitative and quantitative techniques were used, such as interviews and surveys, using stratified random sampling based on the level of income. The results show that $51.3 \%$ of the population is not aware of the environmental and social impacts that are generated by their current consumption habits. The social and environmental problems related to consumption are contamination by domestic garbage, contamination of water sources, excessive use of agrochemicals, food waste and increasing obesity. The population is willing to pay higher prices for products with corporate social responsibility, to reduce food waste, to recycle and reduce food consumption, to contribute to food safety. In conclusion, the population, at present, does not make ethical consumption, causing several social and environmental problems that prevent the achievement of food safety for all inhabitants.
\end{abstract}




\section{INTRODUCCION}

El incremento de la demanda de alimentos debido al rápido crecimiento poblacional trae consigo retos y desafíos para el sector agrícola, como el incremento de la producción y la productividad de los cultivos de manera sostenible, conservando el ambiente a través de prácticas de responsabilidad social empresarial (RSE) de empresas por un lado y por otro a través de un consumo socialmente responsable (CSR) de los consumidores. Al contrario de este planteamiento Carosio (2008), indica que es evidente que la minoría de habitantes de los países industrializados utiliza los recursos planetarios en niveles tales, que ponen en peligro las condiciones que hacen posible la vida, principalmente por sus hábitos de consumo. Como resultado de estas prácticas la consecución de la seguridad alimentaria mundial durante los próximos 40 años requerirá un aumento sostenido de la productividad agrícola (Smyth et al., 2015) y el desarrollo de la Responsabilidad Social Personal (RSP) definido por (López et al., 2017), como la forma en que una persona se desempeña en su vida diaria como miembro de la sociedad y no solo como consumidor, basando sus decisiones en el deseo de minimizar los impactos negativos y maximizar los impactos positivos en los aspectos sociales, ambientales y el entorno económico.

La mayoría de las grandes empresas en la actualidad tienen políticas ambientales, adoptan la teoría del desarrollo sostenible y aspiran a ser corporaciones socialmente responsables. Sin embargo, los críticos sostienen que; incluso el marketing social es una herramienta utilizada por las compañías para no mejorar el bienestar social, sino para lograr una ventaja competitiva con el objetivo de crear riqueza para los propietarios (Roper y Parker, 2013). Desgraciadamente hoy en día las consideraciones que apelan al consumo responsable, $\mathrm{o}$ bien al comercio justo, siguen considerando de alguna manera que la responsabilidad es individual y no eminentemente social (Gil, 2008). En este sentido, (Gjerris et al., 2016) sugieren que la agenda de sostenibilidad debería ser más prominente dentro de la perspectiva ciudadana y que el apoyo al consumismo crítico o ético a través del etiquetado, regulaciones tributarias o prohibiciones de ciertos productos debe ser tomado en mayor consideración. Esto, en definitiva, facilitaría la 'buena' elección por parte de los consumidores, aunque a menudo será una elección de males menores en vez de ideales.

El equilibrio ecológico del planeta es algo que tiene que ver con nuestros niveles de consumo, pero también con los de desecho: estamos dañando ese equilibrio cada vez que consumimos productos que generan grandes cantidades de desechos (como las botellas de plástico, envases no retornables y productos que sólo se usan una sola vez), cuando tiramos al ambiente baterías o no separamos la basura. Todo lo anterior es lo que da una dimensión moral a nuestras acciones de consumo, y en particular al consumismo (Millán, 2012). Así, el consumo es la expresión de la libertad y, por eso mismo, entra por derecho propio en el ámbito ético, en el ámbito de las acciones que se eligen y tiene que ser, por tanto, implícita o explícitamente justificadas (Cortina y Conill, 2009).

Se habla de que las personas tenemos derechos como consumidores, y en los últimos años se ha trabajado al respecto. Sin embargo, se ha relegado el hecho de que, así como tenemos derechos, también tenemos obligaciones o deberes morales, pues con nuestro consumo podemos afectar al medio ambiente, a nuestra comunidad, a nuestra familia, a gente en lugares distantes, e incluso a las generaciones futuras (Millán, 2012). La lucha por los derechos del consumidor no puede ser la lucha de los derechos de un individuo aislado para recibir un producto en buen estado y conforme a lo prometido, sino que debe rebasar en una propuesta de cambio social más general (Gil, 2008). En este sentido (Ariztía et al., 2014) expresa que el consumo ético apenas está despegando. Esto implica también la interacción y el equilibrio de diferentes formas de entender y movilizar la conexión entre el consumo ético, los mercados y las ciudadanías que va de ser entendido principalmente como una fuente de valor económico (como en el contexto corporativo), como un aspecto clave de la ciudadanía derechos (como en el sector de la sociedad civil) o como una dimensión de las políticas gubernamentales (como en el contexto gubernamental).

Uno de los aspectos más relevantes de la ética de consumo tiene que ver con la cantidad de productos y servicios que consumimos en relación con nuestras necesidades, es decir, tiene que ver con el consumismo (Millán, 2012). El imperativo del goce crea una moral emocional y festiva, donde los valores éticos deben su legitimidad a un carácter lúdico de complacencia, ligado a la noción de preferencias, que elimina la distinción entre necesidades y deseos (Carosio, 2008). A su vez, esa capacidad se convierte en la principal fuerza que pone en movimiento a toda la sociedad de consumidores (Lara y Colín, 2007). El consumo llevado al extremo, irreflexivo, irresponsable e irrestricto se transforma en el consumismo o hiperconsumo, el cual resulta perjudicial pues no sólo trastorna a las personas sino que cobra muy caros sus favores trasladando los costos a la mera naturaleza, tanto la interna del ser humano como la externa a éste (González y David, 2009). 
Entendiendo la ética del consumo una buena o mala compra no se basa sólo en conseguir un producto o un servicio de calidad a un buen precio; es necesario que, con nuestro consumo, no dañemos a otros ni al medio ambiente, y que no contribuyamos con situaciones de injusticia social (Millán, 2012). En este sentido Gil (2008), expresa que en el caso de la ética del consumo, nos encontramos con dos posiciones diferentes, una de las cuales considera que a través del consumo responsable las personas podemos cambiar nuestra sociedad, y por otra parte tenemos la posición de quienes consideran que consumir siempre será un mecanismo de perpetuación del capitalismo. Así un consumo ético de las personas involucra la toma de conciencia de los impactos ambientales y sociales que producen nuestros hábitos de consumo en el entorno en que vivimos. También Sudbury y Kohlbacher (2016), coinciden que la mayoría de las definiciones de consumo ético abarcan la referencia a las cuestiones ambientales, cuestiones de justicia social y derechos humanos y por último el tema del bienestar de los animales y de la comunidad local. Es cierto que, en ocasiones, realizar un consumo basado en valores morales puede ser más costoso en términos económicos; pero ese consumo es costoso en la medida en que no hay suficiente oferta dado que no hay suficiente demanda (Millán, 2012).

En este contexto pretendemos relacionar la seguridad alimentaria con un comportamiento ético de consumo de las personas del cantón Santo Domingo de los Colorados tomando conciencia de los impactos ambientales y sociales que se producen por un consumo desmedido, posiblemente limitando el acceso de una parte de la población a los alimentos de la canasta básica familiar poniendo en riesgo su seguridad alimentaria. No se puede decir que el flagelo del hambre se encuentra focalizado en ciertas zonas rurales ya que nada tiene que ver con la escasez de productos alimenticios como es el caso de Santo Domingo de los Colorados. Al respecto (Lara, 2001), indica que la pobreza en nuestro continente ha crecido mucho más en las zonas urbanas que en las rurales durante los últimos 20 años y una de las características más espeluznantes de la estructura agroalimentaria mundial es la coexistencia del hambre con la sobrealimentación que no difiere de la realidad del Ecuador, donde la oferta de alimentos ha sido muy superior al volumen de la población, en promedio el PIB agrícola creció a un 4,9\% frente a un crecimiento poblacional promedio de 1,5\% (Calero, 2011). El mismo autor afirma que la seguridad alimentaria contempla cuatro pilares o dimensiones que son: disponibilidad, acceso, estabilidad, y uso o utilización biológica de los alimentos. Las causas de inseguridad alimentaria pueden ser diversas y multifacéticas, e incluyen factores tales como inestabilidad política, guerras, choques económicos, condiciones climatológicas adversas, desbalances macroeconómicos, degradación ambiental, fenómenos naturales, crecimiento poblacional, pobreza, desigualdad en la distribución de los alimentos al interior de los hogares, inequidad de género, entre otros (Calero, 2011). Varias de estas causas pueden ser el resultado de un consumo sin ética de la población.

Al relacionar el acceso a los alimentos a través del nivel de ingresos por persona se identifica que en el Ecuador para la medición de la pobreza el INEC utiliza recomendaciones internacionales que compara el ingreso per cápita familiar con la línea de pobreza y pobreza extrema que en marzo de 2016 se ubicaron en US\$ 84,25 y US\$ 47,48 mensuales por persona respectivamente. Con este umbral, a nivel nacional la incidencia de la pobreza es del 25,35\% y la extrema pobreza del 10,05\%. A nivel urbano la incidencia de la pobreza es del $16,63 \%$ y la extrema pobreza del 5,60\%, en el área rural la pobreza es del $43,96 \%$ y la pobreza extrema del 19,53\% (Melody Serrano y Serrano, 2016). Esta realidad es una de las causas de la inseguridad alimentaria de un alto porcentaje de la población del país tanto en el sector urbano como rural debido a su bajo poder adquisitivo para acceder a los alimentos. Los medios utilizados por los hogares para adquirir alimentos en Ecuador, en promedio, más del $80 \%$ de los gastos en alimentos proviene de compras y el resto se adquiere mediante otros mecanismos como producción propia, intercambio de alimentos, regalos o donaciones (Calero, 2011). En base a los antecedentes expuestos el objetivo de la presente investigación es determinar como la ética en el consumo de las personas puede contribuir o no a la gestión de la seguridad alimentaria de la población urbana del cantón Santo Domingo de los Colorados.

\section{MATERIALES Y METODOS}

La presente investigación corresponde a un trabajo no experimental bajo un diseño transversal de recolección de datos por medio de entrevistas y la aplicación de encuestas, la técnica a la que más relevancia se la dio es la encuesta, a pesar que también se realizó entrevistas a los gerentes de los principales supermercados y mercados de la ciudad para complementar los resultados. Se empleó un enfoque cualitativo y cuantitativo, en primera instancia se realiza la descripción del fenómeno de estudio y posteriormente un análisis cuantitativo con un alcance de investigación descriptivo correlacional.

El cantón Santo Domingo de los Colorados cuenta con una población de 368.013 habitantes y es la cuarta ciudad más poblada del Ecuador, el tamaño de muestra para esta investigación es de 384 personas, con un nivel de confianza del $95 \%$ y un nivel de significancia del 5\%. Para la aplicación del instrumento de recolección de datos se empleó como marco de muestreo la cabecera cantonal, tomando en consideración 
la clase social en función de los ingresos mensuales de acuerdo a los datos de la Tabla 1, que presenta los resultados de la última Encuesta Nacional de Ingresos y Gastos 2011-2012 de Ecuador cuyos resultados indican que el $54,2 \%$ de la población tienen ingresos menores a dos Salarios Básicos Unificados (528 dólares), el 34,1\% de la población tienen ingresos entre 2 y 5 Salarios Básicos Unificados, y el 11,7\% tienen ingresos mensuales mayores a 5 Salarios Básicos Unificados. Se trabajó con muestro probabilístico, a través del muestreo aleatorio estratificado en función de las clases sociales para obtener una participación representativa de estas, para la recolección de los datos dentro de cada clase social se empleó un muestreo aleatorio simple.

En la codificación se asignó números a las respuestas registradas en cada pregunta del cuestionario que constituyen las variables de estudio. Se aplicó un cuestionario semiestructurado elaborado ad hoc y su diseño contemplo varias fases para incrementar la calidad del instrumento. La encuesta consto de 19 ítems la mayoría con preguntas cerradas de opción múltiple, no se empleó escalas de Likert y se incluyeron las siguientes variables demográficas y socioeconómicas: edad, sexo, nivel de educación y nivel de ingresos mensuales. Además de los aspectos mencionados se estudiaron dos variables: ética del consumo (independiente) y seguridad alimentaria (dependiente). La variable ética del consumo fue explorada atendiendo el nivel de consciencia y percepción de problemas sociales y ambientales de los consumidores del sector urbano del cantón Santo Domingo de los Colorados que se generan por el consumo de bienes y servicios y que afectan la calidad de vida de esta población. Con relación a la variable seguridad alimentaria se exploran las dimensiones de ingreso mensual percibido por las personas de este cantón y el nivel de entendimiento del concepto de seguridad alimentaria, de manera que los ingresos económicos nos permitan categorizar a cada uno de los hogares encuestados como pobres o indigentes con relación a la línea de pobreza, en este caso se usó el nivel de ingreso como variable proxy de la seguridad alimentaria considerando el Enfoque de Economía de los Hogares (EEH).

Con los datos e información recolectada se procedió a realizar el análisis e interpretación por medio del software Statistical Package for the Social Sciences (SPSS).

Tabla 1: Nivel de ingresos. (Encuesta Nacional de Ingresos y Gastos, 2011-2012)

\begin{tabular}{|c|c|c|c|c|}
\hline $\begin{array}{c}\text { Clase } \\
\text { Social }\end{array}$ & $\begin{array}{c}\text { Nivel de } \\
\text { Ingresos }\end{array}$ & $\begin{array}{c}\text { \% de la } \\
\text { Población }\end{array}$ & $\begin{array}{c}\text { Afijación } \\
\text { Proporcional }\end{array}$ & Lugar de Encuestas \\
\hline Baja & $\begin{array}{c}\text { Menor a 2 } \\
\text { SBU }\end{array}$ & $54,2 \%$ & 208 & $\begin{array}{c}\text { Mercados y Ferias } \\
\text { Populares }\end{array}$ \\
\hline Media & $\begin{array}{c}\text { Entre 2 y } 5 \\
\text { SBU }\end{array}$ & $34,1 \%$ & 131 & $\begin{array}{c}\text { Supermercados AKI y } \\
\text { TIA }\end{array}$ \\
\hline Alta & $\begin{array}{c}\text { Más de } 5 \\
\text { SBU }\end{array}$ & $11,7 \%$ & 45 & $\begin{array}{c}\text { Supermaxi (Mega } \\
\text { mercado) }\end{array}$ \\
\hline Total & & & 384 & \\
\hline
\end{tabular}

\section{RESULTADOS}

La exposición de los resultados se divide en 2 partes, primero se describe la zona de estudio y en segundo lugar se presenta el análisis de resultados obtenidos con el análisis estadístico llevado a cabo.

\section{i) Descripción de la zona de estudio}

El cantón Santo Domingo de los Colorados cuenta con una extensión de 3.453,848 $\mathrm{Km}^{2}$, es el sector geográfico donde convergen las provincias de Esmeraldas, Manabí, Guayas, Los Ríos y Cotopaxi, a una altura que va desde 120 m.s.n.m. hasta los 3020 m.s.n.m., y una temperatura promedio de $22^{\circ}$ C. Este cantón cuenta con 8 parroquias: Alluriquín, El Esfuerzo, Luz de América, Puerto Limón, San Jacinto del Búa, Santa María del Toachi, Santo Domingo como parroquia urbana donde vive el 83,05 \% de la población total del cantón que equivale a 305.632 habitantes y por último la parroquia de Valle Hermoso. La mayor densidad poblacional se da en la parroquia urbana con 280,2 habitantes por $\mathrm{Km}^{2}$ en comparación con 14,7 habitantes por $\mathrm{Km}^{2}$ de la parroquia Alluriquín. En el territorio cantonal básicamente se ha desarrollado el sector pecuario que ocupa un $51.01 \%$ de las hectáreas y la agricultura con un $25.58 \%$. Los cultivos con mayor superficie cultivadas son: cacao con un $27.26 \%$, plátano con $24.02 \%$, palma aceitera $20.62 \%$, palmito $7.40 \%$, y yuca con el $4.57 \%$. 
Según datos del INEC 2010, en Santo Domingo de los Colorados el 35,8\% de la población son solteros, el $25,8 \%$ son casados, el $27,7 \%$ son unidos, el $5,8 \%$ son separados, el $3,2 \%$ viudos y el $1,7 \%$ son divorciados. El $32 \%$ de los habitantes de la provincia de Santo Domingo de los Tsáchilas trabaja como empleado privado, el $29,1 \%$ por cuenta propia, el $14,9 \%$ de Jornalero o peón, el $8,4 \%$ del empleado del estado, $4,6 \%$ de patronos, y el $11 \%$ entre trabajador no remunerado, socio y no declarado.

La tasa de crecimiento poblacional intercensal es de 2,36\%, la pobreza de la Zona 4 que incluye a las provincias de Manabí y Santo Domingo de los Tsáchilas, de acuerdo a datos del INEC 2010, es de 60,84\% en el área urbana y $94,70 \%$ en el área rural. La pobreza por Necesidades Básicas Insatisfechas (NBI), por hogar del sector urbano es del 55,55\%, y por personas es del 68,24\%. De acuerdo a la perspectiva que se adopte, la pobreza puede ser medida como la privación al acceso a servicios de salud, educación, servicios básicos, o los bajos niveles de consumo kilo calórico, o la falta de ingresos que permitan acceder a bienes y servicios de mercado (INEC, 2014).

De acuerdo a la Encuesta de Estratificación del Nivel Socioeconómico del año 2011, que considera las dimensiones de vivienda en un $24 \%$, educación en un $17 \%$, económica con $17 \%$, bienes con $16 \%$, tecnológica con un $16 \%$ y hábitos de consumo con 10\%, sobre un gran total de 1000 puntos se dividen los grupos socioecomicos del Ecuador de la siguiente manera desde un nivel superior al inferior, grupo A de 845 a1000 puntos, grupo B de 696 a 845 puntos, grupo $\mathrm{C}+$ de 535 a 696 puntos, grupo C- de 316 a 535 puntos y grupo D de 0 a 316 puntos. En la clase $A$ se encuentra el 1,9\% de la población, el 11,2\% en la clase $B$, el $22,8 \%$ en la clase $\mathrm{C}+$, el $49,3 \%$ en la clase $\mathrm{C}-\mathrm{y}$ el $14,9 \%$ en la clase D.

El cantón Santo Domingo de los Colorados se caracteriza por la diversidad cultural, ya que sus habitantes son originarios de deferentes regiones del Ecuador y es considerada como la cuarta ciudad más poblada del país. La diferencia entre clases sociales en función del ingreso y los hábitos de consumo es marcada, ya que la población de clase A realiza las compras de alimentos en Supermercados y accede a productos con garantía de calidad y de marcas reconocidas que cubren sus necesidades y expectativas. Mientras que por el otro lado existe una gran mayoría de la población que tiene menores ingresos y trabaja de manera informal para "ganar su semana", sus hábitos de consumo se remiten únicamente a la compra en la tienda de barrio por unidades o porciones para el consumo diario de su familia.

\section{ii) Desarrollo}

Es importante resaltar que la presente investigación es un estudio de percepciones por encuesta estratificada socialmente de la población urbana del cantón de Santo Domingo de los Colorados que analiza la relación existente entre seguridad alimentaria como variable dependiente y la ética del consumo más una serie de variables socioeconómicas y de percepción como variables independientes a través de un modelo de regresión lineal múltiple para explicar dicha relación. En el procesamiento estadístico de las variables cualitativas como medida de resumen se emplearon las frecuencias relativas y tablas de contingencia, considerando el alcance descriptivo correlacional de la investigación. Para definir el grado de asociación de las variables independientes con la variable proxy de seguridad alimentaria se consideró el nivel de significancia critico (alpha $<0,05$ ) obtenido a través de la regresión múltiple, para identificar las variables significativas estadísticamente que contribuyen a garantizar la seguridad alimentaria de la población urbana de este cantón.

La población encuestada está compuesta por el 40,5\% de personas del género masculino y 59,5\% del género femenino. La edad promedio de los encuestados es de 35,5 años con un rango de edad de 18 a 80 años. El 17,2\% de los encuestados tiene un nivel de educación primario, el 39,2\% tiene un nivel de educación secundaria, predominantemente el 42,6\% poseen educación universitaria y únicamente el $1 \%$ tiene un nivel de educación de posgrado.

Más de un tercio de la población (34,4\%) encuestada perciben ingresos mensuales menores a 300 dólares, el 38,7\% perciben ingresos en un rango de 301 a 600 dólares, el 16,9\% entre 601 y 900 dólares y únicamente el $10 \%$ de la población tiene ingresos mayores a 900 dólares por mes. Tomando como referencia que el costo de la canasta familiar básica del Ecuador en agosto del 2017 se ubicó en 708,79 dólares podemos decir que $65,6 \%$ de la población urbana de Santo Domingo de los Colorados con sus ingresos actuales no cubre las necesidades básicas de alimentación poniendo en riegos la seguridad alimentaria personal y el de su familia por falta de poder adquisitivo para acceder a los alimentos en cantidad y calidad todo el tiempo. No existe correlación entre el nivel de ingresos y un comportamiento de consumo ético. En este mismo sentido al relacionar el conocimiento de la población sobre el concepto de seguridad alimentaria el $41,5 \%$ de la población desconoce que es la seguridad alimentaria y el $58,5 \%$ de la población señalan que entienden este concepto. También sorprendentemente $82,8 \%$ de la población encuestada manifiesta la intención de reducir el consumo de bienes y servicios para contribuir a alcanzar la 
seguridad alimentaria y nutricional de la población de este cantón. Se determinó una correlación positiva altamente significativa $(p<0,00)$, entre la ética del consumo y el entendimiento del concepto de seguridad alimentaria, ya que el $68,5 \%$ de las personas que entienden el concepto de seguridad alimentaria afirman que tienen conciencia de los impactos de sus hábitos de consumo, por el contrario el 64,9\% de las personas que no conocen que es la seguridad alimentaria tampoco son conscientes de los impactos ambientales y sociales que producen con el consumo.

Con relación a la variable de estudio ética del consumo, entendiendo la misma como el nivel de conciencia de los impactos ambientales y sociales que producimos al momento de adquirir bienes y servicios para nuestro consumo, el $48,7 \%$ de la población encuestada manifiesta que es consciente de los impactos que se generan con el consumo, mientras que el $51,3 \%$ de la población no tiene conciencia de los impactos directos de sus hábitos de consumo. Por otro lado sorprendentemente el $93,1 \%$ de los encuestados manifiestan que perciben problemas ambientales y sociales debido a un consumo irrestrictito; lo que se contrapone con los resultados del nivel de conciencia de los impactos ambientales y sociales derivados del consumo detectados en este cantón, esto se debe a que todos los habitantes sean conscientes o no de los impactos del consumo son afectados por este tipo de problemas como sociedad en general. El 90,8\% de la población manifiesta que las empresas deberían producir de manera sostenible a través de prácticas de responsabilidad social empresarial (RSE) y así cumplir con su verdadero rol para contribuir a minimizar este tipo de problemas derivados del consumo sin ética. Estos hallazgos son muy valiosos ya que más de la mitad de los habitantes de este cantón no son conscientes de los impactos ambientales y sociales que producen con sus hábitos de consumo sin embargo un considerable porcentaje de población se ven afectados por este tipo de problemas de manera directa, afectando su calidad de vida sin embargo aún no asumen sus responsabilidades y delegan a las empresas la tarea de reducir este tipo de problemas a través de prácticas de Responsabilidad Empresarial Corporativa. No existe correlación significativa entre el sexo de las personas con la actitud hacia un comportamiento de consumo ético.

En este mismo sentido el 57,9\% de la población encuestada expresa no conocer que es un consumidor socialmente responsable, lo que explica en gran medida por que la mayoría de la población no realiza un consumo ético que se complementen con las iniciativas de RSE de la empresas locales para minimizar los impactos negativos derivados del consumo. Se detectó una correlación negativa $(p<0,04)$ entre la ética del consumo y el nivel de educación de las personas. Al analizar los principales problemas ambientales derivados del consumo que afectan a la población de Santo Domingo, el 32,6\% de los encuestados indican que la contaminación con basura doméstica y falta de reciclaje y clasificación de basura como el más importante, el $29 \%$ mencionan a la contaminación de las fuentes naturales de agua como otro problema ambiental, el 16,2\% señalan al uso excesivo de agroquímicos, la contaminación por la industria y vehículos es percibida por el $11,5 \%$ de la población, y el 10,3\% considera la deforestación también como un problema ambiental relacionado con el consumo entre los principales. Desde el punto de vista de los problemas sociales que se perciben en el cantón Santo Domingo de los Colorados en primer lugar resalta el desperdicio de alimentos en los hogares considerado por el 37,4\% de la población, el 26,4\% manifiestan que la obesidad de las personas asociada al consumo es el segundo problema de importancia, y un 16,5\% de la población considera un problema social al sobreendeudamiento que está muy relacionado con los hábitos de consumo de productos suntuarios para la satisfacción de deseos y las facilidades de pago brindadas por las empresas fabricantes.

Un resultado a resaltar es que el $64,4 \%$ de la población estaría dispuesta a pagar un precio más alto por productos y servicios que sean producidos con RSE, esto se relaciona con la alta percepción de los problemas ambientales y sociales que afectan la calidad de vida de sus habitantes y que buscan una solución a través de prácticas de Responsabilidad social empresarial por parte de las empresas del cantón. Los habitantes encuestados expresan que podrían contribuir a la reducción de los problemas ambientales y sociales ligados al consumo, a través de la disminución el desperdicio de alimentos expresado por el $40 \%$ de la población, el 34,9\% de la población a través prácticas de reciclaje y clasificación de basura, y únicamente el $11,8 \%$ mediante la reducción del consumo de bienes y servicios. En esta misma línea el $78,7 \%$ de la población estarían dispuestos en dejar su vehículo en casa para disminuir la contaminación del aire, y optar por usar otros medios de transporte como la bicicleta o el autobús.

Por otro lado los resultados indican que el $45,4 \%$ de la población prioriza la calidad de los productos como factor decisivo de compra, el 15,9\% el factor salud, el 15,6\% consideran que los productos sean amigables con el ambiente y sorprendentemente el precio no es un factor importante de decisión de compra de bienes y servicios ya que solo lo mencionan el $13,6 \%$ de los encuestados. Este resultado complementa la disposición de la población a pagar un precio más elevado por productos elaborados con responsabilidad social empresarial que contribuyan a la reducción de los problemas ambientales y sociales relacionados con el consumo. El 46,2\% de la población de este cantón compra sus alimentos en supermercados, el 36,4\% lo realiza en ferias populares, el $10 \%$ de la población en tiendas de barrio y solo el 5,4\% los compra por 
internet. No existe correlación significativa entre la clase social y el lugar de compra, ya que el $43,3 \%$ de la población de clase baja también accede a los supermercados, mientras que únicamente el $4,2 \%$ de la población de clase alta compra las ferias populares. Por otro lado el $84,1 \%$ de la población expresa que la publicidad y marketing desarrollada por las empresas deberían informar las desventajas ecológicas que producirían el consumo de sus productos para ayudar en la toma de decisiones conscientes por parte de los consumidores.

Otro importante resultado obtenido en la presente investigación es que el 70,5\% de la población no identifica a empresas locales que apliquen RSE en sus sistemas de producción, únicamente resaltan empresas con alcance nacional como Pronaca con el 9\% de frecuencia, Coca Cola con el 2,6\%, La Fabril con el $2,3 \%$ y Supermaxi con 2,1\%. Estos resultados probablemente hacen referencia a empresas que cuentan con unidades de producción en este cantón, lo que evidencia la falta de comunicación y difusión a la sociedad en general de las prácticas de responsabilidad social empresarial que desarrollan las empresas en este cantón y no contribuyen de esta manera a impulsar o desarrollar una actitud de consumo ético de sus habitantes. Para contrastar la Hipótesis nula $(\mathrm{H} 0)$, que sugiere que a través de un consumo ético medido a través del nivel de concienciación de los impactos ambientales y sociales que se producen por el consumo de las personas no se puede contribuir de manera importante a la gestión de la seguridad alimentaria de la población del cantón Santo Domingo de los Colorados, se seleccionó el análisis de regresión lineal múltiple como el método estadístico para analizar las relaciones entre las variables de estudio.

Tabla 2: Estadística de regresión

\begin{tabular}{|l|l|}
\hline Coeficiente de correlación múltiple & 0.434 \\
\hline Coeficiente de determinación $\mathrm{R}^{2}$ & 0.188 \\
\hline $\mathrm{R}^{2}$ ajustado & 0.176 \\
\hline Error Típico & 0.959 \\
\hline
\end{tabular}

De acuerdo a los resultados obtenidos en el análisis de regresión y presentados en la Tabla 2, a través del coeficiente de determinación ajustado $\left(R^{2}\right)$ podemos decir que el $17.6 \%$ de la variación de la seguridad alimentaria por falta de acceso a los alimentos medida a través del nivel de ingresos mensuales de la población, está determinada de manera significativa $(p<0,01)$ por las variables edad, clase social, sexo y el nivel de educación alcanzado por su población. En tanto que la Ética del consumo de las personas medida a través del nivel de concienciación y percepción de los problemas ambientales y sociales que se producen por el consumo no presentan un efecto significativo sobre la gestión de la seguridad alimentaria, es decir la población actual no está sensibilizada hacia un consumo consciente o crítico y tampoco relaciona los problemas ambientales y sociales que se presentan en esta ciudad con los hábitos de consumo de su población. Con estos resultados aceptamos la Hipótesis nula, ya que la población de Santo Domingo de los colorados no tiene un consumo ético por falta de conciencia de los impactos ambientales y sociales que se producen con los hábitos de consumo actual y de esta manera no podemos contribuir actualmente a la gestión de la seguridad alimentaria.

Tabla 3: Modelo de regresión

\begin{tabular}{|c|c|c|c|c|c|}
\hline & $\begin{array}{c}\text { Suma de } \\
\text { cuadrados }\end{array}$ & $\begin{array}{c}\text { Grados de } \\
\text { libertad }\end{array}$ & Media cuadrática & $F$ & Sig. \\
\hline Regresión & 81.832 & 6 & 13.639 & 14.822 & 0.000 \\
\hline Residual & 352.434 & 383 & 0.920 & & \\
\hline Total & 434.267 & 389 & & & \\
\hline
\end{tabular}

El modelo de regresión indica que existe un efecto altamente significativo para las variables referidas, dado que se obtuvo un $\mathrm{p}<0,000$ presentado en la Tabla 3 , el cual es menor que el nivel crítico de comparación (alpha $\leq 0,05$ ).

Como se puede observar en los coeficientes estandarizados (Beta) en la Tabla 4, las variables Edad y Nivel de Educación tienen un efecto positivo y significativo sobre la variable dependiente nivel de Ingresos como variable proxy de la seguridad alimentaria. Con relación a las variables Clase Social y Sexo que también presentan un efecto significativo sobre el nivel de Ingresos, los coeficientes estandarizados Beta, presentan un signo negativo que indica que las mujeres perciben menores ingresos que los hombres y que existe una diferencia de ingresos a favor de las clases sociales más altas con relación a las clases sociales más bajas. 
Tabla 4: Coeficientes Estadísticos

\begin{tabular}{|l|c|l|l|l|l|}
\hline \multirow{2}{*}{ Modelo } & \multicolumn{2}{c|}{$\begin{array}{c}\text { Coeficientes no } \\
\text { estandarizados }\end{array}$} & $\begin{array}{c}\text { Coeficientes } \\
\text { tipificados }\end{array}$ & \multirow{2}{*}{$t$} & \multirow{2}{*}{ Sig. } \\
\cline { 2 - 5 } & $B$ & Error típico & Beta & & \\
\hline (Constante) & 1.816 & 0.464 & & 3.912 & 0.000 \\
\hline Edad & 0.024 & 0.004 & 0.293 & 5.826 & 0.000 \\
\hline Clase Social & -0.212 & 0.069 & -0.142 & -3.060 & 0.002 \\
\hline Sexo & -0.452 & 0.100 & -0.210 & -4.523 & 0.000 \\
\hline Nivel de Educación & 0.401 & 0.071 & 0.285 & 5.626 & 0.000 \\
\hline Ética del Consumo & -0.070 & 0.098 & -0.033 & -0.717 & 0.474 \\
\hline $\begin{array}{l}\text { Percepción } \\
\begin{array}{l}\text { Problemas Sociales y de } \\
\text { Ambientales }\end{array}\end{array}$ & -0.164 & 0.195 & -0.039 & -0.839 & 0.402 \\
\hline
\end{tabular}

\section{DISCUSION}

Para iniciar un consumo ético el primer paso es querer consumir éticamente; el segundo, hacer conciencia personal; y el tercero, compartir la experiencia con los demás, para construir una conciencia colectiva crítica. A la hora de comprar se debe analizar además de la utilidad individual y el precio si realmente se necesita ese producto. Probablemente se lo puede reemplazar o incluso prescindir de él, lo cual reducirá el consumismo y beneficiará al presupuesto familiar. Es fundamental considerar el número de veces que se usará ese producto y qué se hará con él (y con su envase) cuando se lo deseche. Se debe pensar cuánto contaminará. El consumo ético implica buscar información con un pensamiento crítico de la realidad, de la publicidad y de los medios de comunicación, para poder cuestionar lo que está detrás de lo que se consume y saber cuáles son sus consecuencias (Sotorno, 2007).

En los países en desarrollo, a menudo más de la mitad de los ingresos de los hogares se gastan en alimentos, por lo que los pobres son altamente vulnerables a fluctuaciones repentinas de precios que pueden conducir a la gente a la pobreza (Neira et al., 2013). En el caso del Ecuador, los problemas alimenticios no se ligarían a una insuficiente disponibilidad de alimentos, sino a la dificultad de las personas/hogares en acceder a una canasta de alimentos que satisfaga las necesidades básicas, ya que, una proporción de hogares no cuenta con recursos económicos para acceder a una cantidad mínima de alimentos (Calero, 2011). Estos resultados coinciden con nuestros hallazgos sobre seguridad alimentaria medido a través del nivel de ingreso que indican que el $65,6 \%$ de la población de estudio tienen ingresos mensuales menores al costo de la canasta básica mensual del Ecuador, lo que no les permitiría cubrir sus necesidades básicas de alimentación, poniendo en riesgo la seguridad alimentaria por falta de acceso a los alimentos. En este sentido de acuerdo (Sanchez, 2018), en su investigación realizada en el sector rural de Santo Domingo de los Colorados indica que de alguna manera, establecen un perfil de la población cuya seguridad alimentaria se encuentra en riesgo, contribuyendo al diseño e implementación de políticas alimentarias que garanticen el acceso a una alimentación adecuada a las poblaciones rurales a través de la agricultura familiar.

Nuestros resultados también muestran que más de la mitad de la población del cantón Santo Domingo de los Colorados afirman que conocen el concepto de seguridad alimentaria y que hace un uso racional de los alimentos disponibles. En este contexto Dou et al., (2016), indican que la seguridad alimentaria depende del uso sostenible de los recursos naturales como la tierra, el agua, los nutrientes, los combustibles fósiles, etc. Siguiendo este enfoque es importante relacionar el nivel de ingresos con el cambio de hábitos de consumo de la población, ya que los consumidores a nivel mundial han cambiado de considerar la proteína animal un artículo de lujo en su alimentación a ser una parte regular de su dieta por ejemplo. Por lo tanto, el aumento de los precios de los alimentos puede influir en el bienestar del consumidor no sólo por afectar la calidad y la cantidad de alimentos asequibles, sino también por el impacto negativo de la economía en general (Regmi y Meade, 2013). La población pobre enfrenta limitaciones tanto para acceder al mercado como para producir sus propios alimentos, lo que los hace más susceptibles de sufrir problemas alimenticios (Calero, 2011). La dimensión ética y política del consumo constituye una preocupación creciente para capas de la población que empiezan a considerar su responsabilidad, y su poder, como consumidores (Feenstra, 2015).

Más de la mitad de la población del cantón Santo Domingo de los Colorados afirman no ser conscientes de los impactos ambientales y sociales que generan con sus hábitos de consumo, no se encontró correlación entre la edad de las personas y la actitud hacia un consumo ético que minimicen los impactos ambientales y 
sociales del consumo. La educación presenta una correlación negativa significativa $(p<0,04)$, con la variable ética del consumo ya que conforme se incrementa el nivel de educación también se incrementa el nivel de ingresos $(p<0,00)$ con lo que cambian sus hábitos de consumo y baja el nivel de conciencia de los impactos ambientales y sociales asociados al consumo. Este resultado difiere con lo expuesto por (Johnston et al., 2011) que indican que los estudiosos entienden el consumo ético como una forma clave en que los individuos pueden abordar los problemas sociales y ecológicos. Si bien es una tendencia esperanzadora, plantea la pregunta de si el consumo ético es principalmente una práctica social de élite, especialmente dado que se cree que los nichos de mercado de productos alimenticios éticos (por ejemplo, orgánicos, comercio justo) atraen a consumidores ricos y educados. Tampoco se encontró correlación entre las variables clase social y sexo, con la ética del consumo de la población del cantón Santo Domingo de los Colorados. Estos resultados concuerdan con lo expuesto por (Saad et al., 2009) que indican que el comportamiento económico de las personas varía según la etapa del ciclo de vida en la que se encuentran, los cambios en la estructura por edades tienden a producir un impacto importante sobre el proceso de desarrollo económico y por ende de sus hábitos de consumo. Así se considera, en efecto, la producción, tenencia, uso y consumo de bienes como un valor central de nuestra sociedad actual (Argandoña, 2003).

Al relacionar la ética del consumo con la percepción de problemas ambientales y sociales como efecto del consumo irrestricto de las personas de esta localidad identificamos que no existe ningún tipo de correlación, lo que ayuda a la comprensión y descripción de resultados, ya que por un lado más de la mitad de la población afirma no tener conciencia de los problemas ambientales y sociales relacionados con el consumo (ética de consumo) mientras que el 93,1\% de esta misma población menciona que percibe o son afectados por este tipo de problemas y que afectan su calidad de vida. Es decir la población local no asume su responsabilidad y trasladan estos efectos a las empresas productoras de bienes y servicios y al estado para que apliquen un enfoque de RSE como mecanismo de solución de los problemas sociales y ambientales del consumo. En este sentido una teoría normativa de consumo se hace necesaria, una teoría acorde con esa noción de ciudadanía densa como es el caso de Santo Domingo de los Colorados. Pero para eso hace falta, en primer lugar, tratar de comprender ese misterioso mundo del consumo, saber si el nivel de consumo de las personas depende del nivel de ingreso; si hay otras metas del consumo además de la supervivencia; si la única motivación para consumir de una manera compulsiva, que es el gran mal de la época, es el afán de emulación, si no hay otras motivaciones muy comprensibles para la carrera trabajo-producción-consumo-trabajo (Cortina, 2002). Curiosamente, ha habido poco escrito, por lo menos explícitamente, sobre la ética de lo que comemos y los valores que queremos mantener para un sistema alimentario más sano, sostenible y global. En este sentido la mayor parte de los enfoques de las investigaciones han sido sobre aspectos de la manera injusta en que se produce nuestra comida, sobre las desigualdades en el acceso a alimentos nutritivos de alta calidad y en las opciones de alimentos dejando de lado la ética del consumo (Fanzo, 2015).

La percepción de gran parte de la población de Santo Domingo de Colorados indica que el mayor problema social debido a un consumo sin conciencia es el desperdicio de alimentos en los hogares y en centros comerciales de la ciudad, lo que coincide con los resultados reportados por Dou et al., (2016), que indica que la reducción del desperdicio de alimentos es una de las estrategias clave para combatir el hambre y alimentar de manera sostenible al mundo, indicando que Estados Unidos pierde al menos 150 millones de toneladas métricas (MMT) entre la granja y el tenedor anualmente, de los cuales alrededor de 70MMT es la pérdida de alimentos comestibles. Mejorar el uso de lo que ya se produce reduciendo el desperdicio merece atención en el esfuerzo por abordar el creciente desafío de la seguridad alimentaria. Una mayor eficiencia de utilización de alimentos mediante la reducción de los residuos también contribuiría a mejorar la resistencia de los sistemas alimentarios locales y mundiales, que es cada vez más importante ante el cambio climático. En este sentido (HLPE, 2014), indica que el problema de los residuos de consumo es principalmente un problema en los países desarrollados. Sin embargo, las economías emergentes se enfrentan cada vez más a un desafío similar: el crecimiento de los ingresos y los cambios demográficos de los últimos 20 años han traído consigo un cambio en los hábitos alimenticios con una explosión en el consumo de alimentos procesados y una convergencia relativa de dietas, el surgimiento de problemas de obesidad, aumentando rápidamente incluso en algunos casos entre la parte más pobre de la población. Este cambio también tiene consecuencias para la sociedad, pues la forma en que un individuo se comporta en el consumo, es una expresión del grupo social, las normas y patentes culturales al que se quiere pertenecer (Lugo, 2010). En definitiva los alimentos desperdiciados son recursos desperdiciados (Dou et al., 2016).

En la actualidad, casi 1.000 millones de personas en el mundo padecen hambre o desnutrición, mientras que 1.500 millones de personas tienen sobrepeso u obesidad (FAO, 2012). En nuestros resultados la obesidad de las personas es considerada como el segundo problema de importancia social asociado a un consumo sin ética que presenta una correlación negativa significativa $(p<0,02)$ entre las personas que afirman tener un consumo ético y la percepción de la presencia de problemas sociales y ambientales relacionados con el consumo, es decir la población de este cantón como se explicó anteriormente no realiza 
un consumo ético en su vida cotidiana. Al respecto Serrano (2012), postula que en Ecuador existen nuevos patrones de sobrepeso y obesidad, que afectan sobre todo a mujeres, muchas de ellas pertenecientes a las clases socio-económicas menos favorecidas, lo que se debería, entre otras cosas, a una transición nutricional, que consiste en la sustitución de una dieta baja en calorías, dependiente de un predominio de calorías de origen vegetal, por otra hipercalóricos, dependiente de alimentos de origen animal (grasas) y también de azúcares refinadas. A nivel país y en Santo Domingo de los Colorados se comercializan actualmente alimentos procesados regidos por la norma de etiquetado de alimentos, esta norma aplica a productos nacionales e importados. En este sistema de etiquetado si identifica si los productos son altos, medios o bajos en el contenido de azúcar, grasa y sal para que la decisión de su consumo recaiga en el ciudadano plenamente informado.

Los principales problemas ambientales asociados al consumo percibido en la población del cantón Santo Domingo de los Colorados en orden de importancia son la contaminación con basura doméstica, la contaminación de las fuentes naturales de agua, uso excesivo de agroquímicos, contaminación vehicular e industrial y la deforestación. En este punto es importante señalar que el concepto de espacio ambiental ilustra que se debe superar el subconsumo socialmente insostenible y que se debe eliminar el sobreconsumo ambientalmente insostenible. El fuerte consumo sostenible requiere un cambio institucional (Spangenberg, 2014). Nuestros resultados concuerdan con lo expuesto por (Garnett, 2014) que menciona que el sistema alimentario de hoy está destruyendo el medio ambiente del que depende la producción de alimentos en el futuro. Contribuye a un $20-30 \%$ de las emisiones antropogénicas de gases de efecto invernadero (GEI), es la principal causa de deforestación, cambio en el uso de la tierra y pérdida de biodiversidad; representa el $70 \%$ de todo el uso humano de agua y es una fuente importante de contaminación del agua. Por otro lado un axioma que ha dado forma a los enfoques de política para el consumo sostenible ha sido que si más consumidores entienden las consecuencias ambientales de sus patrones de consumo, a través de sus elecciones de mercado inevitablemente presionarían a los minoristas y fabricantes para avanzar hacia la producción sostenible (Akenji, 2014). De acuerdo a (Sampaio, 2009), indica que el sistema de producción que recurre a la siembra directa, demostró tener, no solo un potencial de conservación, sino también, un potencial de recuperación de este recurso. Esto representa una importante alternativa cuanto a la reducción de procesos graves de degradación medioambiental inducidos por la agricultura intensiva.

Más de la mitad de la población de estudio de Santo Domingo de los Colorados estaría dispuesta a pagar un precio más alto por productos y servicios que sean producidos con responsabilidad social empresarial, ya que se ven afectados de manera directa por los problemas ambientales y sociales derivados del consumo sobre la calidad de vida. En este sentido Rivera et al., (2016), muestran una relación directa positiva entre la responsabilidad social empresarial (RSE) y la satisfacción del cliente con formación en RSE y las iniciativas ambientales. También (Vermeir y Verbeke, 2008) mencionan que un número substancial de estudios muestran que los consumidores valoran el aspecto ético de un producto y que existe la voluntad de pagar por productos éticos. Sin embargo, la intención de comportamiento de los consumidores en el mercado aparentemente no es coherente con su actitud informada hacia los productos con una dimensión ética. Un resultado que aporta evidencia a la falta de consumo ético de las personas de Santo Domingo de los Colorados indica que la mitad de la población, afirman no conocer lo que es un consumidor socialmente responsable (CSR).

El consumo responsable se define como la elección de productos basados no sólo en la relación calidadprecio, sino también en los impactos sociales y ambientales de los productos y servicios, así como en la buena gobernanza de las organizaciones que los ofrecen. Se afirma que el consumidor socialmente responsable (CSR) es uno de los principales impulsores de la RSE (López et al., 2017). En particular, podemos extraer que aquellas empresas que orienten sus actuaciones de RSE hacia el cliente conseguirán generar un doble efecto. Por una parte lograrán mejorar la actitud hacia la empresa, y lo que es mejor, incrementar la intención de compra futura de sus productos frente a otros que provengan de empresas que no atiendan suficientemente estás responsabilidades (Parada et al., 2014). En este sentido Marquina y Reficco (2012), indican que la probabilidad de compra de los consumidores de zapatillas aumenta si la empresa fabricante cumple con determinadas características en su gestión: compromiso con el medio ambiente, buen trato a los trabajadores, apoyo a programas de lucha contra la pobreza, calidad de los productos y oferta de productos atractivos e innovadores. Al respecto (Arredondo et al., 2011), indican que los organismos defensores de los derechos de los consumidores están más atentos de exigir a las empresas que incluyen a la RSE, proporcionar la información veraz al consumidor sobre sus prácticas de RSE, ya que éste es un criterio que está siendo considerado en la toma de decisiones del cliente consumidor.

De acuerdo a López et al., (2017), indican que la responsabilidad social empresarial no puede ser una realidad sin una evolución paralela de comportamientos responsables individuales, alineados con las 
pretensas premisas y valores que están ganando espacio en los campos social, ambiental y económico. Es así que cconsideramos oportuno mencionar que más de dos terceras partes de la población encuestada no reconocen a empresas locales o nacionales que desarrollen prácticas de RSE en sus actividades de producción, en el cantón Santo Domingo de los Colorados. Este hallazgo se constituye en una potencial oportunidad para desarrollar estrategias de comunicación y difusión de las practicas RSE que llevan a cabo las empresas.

Con la finalidad de analizar los hábitos de consumo de la población de estudio y como se relacionan estos con la ética del consumo se destacan como resultados a los principales factores de influencia en la decisión de compra a la calidad del producto, el aporte a la salud del consumidor y la percepción de que sean productos amigables con el ambiente. En este sentido los participantes del consumo consciente muestran flexibilidad en las elecciones éticas y no éticas de compra "dependiendo de su propio estado de ánimo y factores como el precio, la calidad, la comodidad, el gusto y los deseos de otros cercanos a ellos (Carrington, Neville, y Whitwell, 2014). Los principales lugares de compra de alimentos de esta población son los supermercados, las ferias populares y las tiendas de barrio. Esta información podría ser de gran utilidad en las campañas de comunicación de las prácticas de responsabilidad social empresarial que llevan a cabo las empresas productoras de bienes y servicios del cantón y del país. Al respecto Millán (2012), indica que con frecuencia a la publicidad se le acusa de ser engañosa, de mentir, de ser injusta, de presentar estereotipos (raciales, sexuales, sociales), de fomentar el materialismo y el consumismo, etc., es decir, de fomentar el consumo de productos que no necesitamos. La información es entonces el elemento fundamental para dar a conocer los productos y servicios que se ofertan en el mercado y poner a disposición del consumidor la diversidad de opciones de consumo disponibles para que decida cuáles son mejores, esto es, cuales satisfacen sus necesidades y no afectan su salud (Lugo, 2010). En este sentidos por ejemplo las tradiciones francesas de moderación (frente a la abundancia estadounidense), se centran en la calidad (frente a la cantidad) y se centran en las alegrías del momento (en lugar de hacer que la vida sea cómoda y fácil) respaldan un estilo de vida más saludable. El entorno físico francés fomenta edades tempranas, alimentación social moderada, meriendas mínimas y más actividad física en la vida diaria como medidas de consumo ético y responsable (Rozin, 2005). El consumo ético también implica reducir los niveles y malos hábitos de consumo, de manera que sea posible optar por un modelo de bienestar no basado en los bienes materiales. Si cambian las prioridades se puede dedicar una mayor parte del presupuesto a la alimentación saludable, a disfrutar el tiempo libre de una manera más constructiva y solidaria (Sotorno, 2007).

Como posibles cambios de actitud en el comportamiento de la población de este cantón y como mecanismos que contribuyan en la solución de los problemas ambientales y sociales por efectos del consumo proponen reducir el desperdicio de alimentos, reciclar los desechos generados en los hogares y disminuir el consumo a través de una compra consciente. Estos resultados son una señal de cambio que deben ser apoyados por las entidades del estado y la sociedad en general para crear consumidores responsables y asuman la responsabilidad social personal para cumplir su rol como ciudadano y no únicamente como consumidor. Estos posibles cambios se relacionan con la definición de consumo ético de Barnett et al, citado por (Adams y Raisborough, 2010), que refiere que el consumo ético es "cualquier práctica de consumo en la que registrar explícitamente el compromiso con otros distantes o ausentes es una dimensión importante del significado de la actividad de los actores involucrados". Un ejemplo de este tipo cambios es mencionado por (Peattie y Peattie, 2009) que indica que las campañas contra el tabaquismo, como la campaña de la verdad, han hecho un trabajo notable al reposicionar el hábito de fumar de una norma social a algo ampliamente inaceptable y pasado de moda en muchos países, lo que puede suceder en Santo Domingo de los Colorados si encontramos los mecanismos para convertir las actitudes en comportamientos. Sería de gran importancia plantear nuevas investigaciones que complementen nuestros hallazgos en temas como nivel de desperdicio de alimentos en las familias, identificar las razones de la falta de ética en el consumo y conocer el rol del estado en la contribución de la resolución de problemas ambientales y sociales derivados de un consumo sin consciencia ni ética alguna.

\section{CONCLUSIONES}

Los resultados reflejan que la población del cantón Santo Domingo de los Colorados no son conscientes de los impactos ambientales y sociales que se generan con los hábitos de consumo actuales, es decir no podemos hablar de una población que tiene consumo ético, por ende no contribuye en la gestión de la seguridad alimentaria.

Un resultado importante indica que esta población está dispuesta a pagar un precio más alto por bienes y servicios producidos con responsabilidad social empresarial como mecanismo para minimizar los problemas ambientales y sociales derivados del consumo que afectan la calidad de vida de la mayoría de personas que viven en este cantón. 
Los problemas ambientales y sociales más importantes que afectan la calidad de vida de esta población que están relacionados con el consumo son la contaminación por basura doméstica, contaminación de las fuentes de agua, uso excesivo de agroquímicos, desperdicio de alimentos y el incremento de los índices de obesidad.

Existe una gran oportunidad de crecimiento sostenible para las empresas que desarrollan prácticas de responsabilidad social empresarial en sus actividades productivas o de servicios ya que la población se ve sensibilizada por los problemas sociales y ambientales derivados del consumo.

\section{REFERENCIAS}

Adams, M. y J. Raisborough, Making a Difference: Ethical Consumption and the Everyday, https://doi.org/10.1111/j.1468-4446.2010.01312.x, British Journal of Sociology, 61(2), 256-274 (2010)

Akenji, L., Consumer Scapegoatism and Limits to Green Consumerism, https://doi.org/10.1016/j.jclepro.2013.05.022, Journal of Cleaner Production, 63, 13-23 (2014)

Argandoña, A., La Ética de la Sociedad de Consumo, Cuadernos Empresa y Humanismo, (37), 1-32 (2003)

Ariztía, T., D. Kleine y otros cuatro autores, Ethical Consumption in Brazil and Chile: Institutional Contexts and Development Trajectories, https://doi.org/10.1016/j.jclepro.2013.04.040, Journal of Cleaner Production, 63, 84-92 (2014)

Arredondo, G., V. Maldonado y J. De La Garza, El Consumidor Ante la Responsabilidad Social Empresarial, Actitudes según Edad y Género, Cuadernos de Administración, Classificação JEL: M10, M14, 24(43), 285-305 (2011)

Calero, C., Seguridad Alimentaria en Ecuador desde un Enfoque de Acceso a Alimentos, FLACSO-Ecuador, 1-111 (2011)

Carosio, A., El Consumo en la Encrucijada Ética, Utopía y Praxis Latinoamericana, 41, 13-45 (2008)

Carrington, M.J., B.A. Neville y G.J. Whitwell, Lost in translation: Exploring the Ethical Consumer Intention-Behavior Gap, https://doi.org/10.1016/j.jbusres.2012.09.022, Journal of Business Research, 67(1), 2759-2767 (2014)

Cortina, A., Por una Ética del Consumo, 1a Ed., 1-353, Santillana Ediciones Generales, Madrid, España (2002)

Cortina, A. y J. Conill, La Responsabilidad Ética De La Sociedad Civil, Mediterráneo Económico, 26, 13-29 (2009)

Dou, Z., J. Ferguson y otros cuatro autores, Assessing U.S. Food Wastage and Opportunities for Reduction, https://doi.org/10.1016/j.gfs.2016.02.001, Global Food Security, 8, 19-26 (2016)

Fanzo, J., Ethical Issues for Human Nutrition in the Context of Global Food Security and Sustainable Development, https://doi.org/10.1016/j.gfs.2015.11.00, Global Food Security, 7, 15-23 (2015)

Feenstra, R. A., El Consumo Ëtico ¿Una Nueva Forma de Participación Ciudadana? 1-18 (2015)

Garnett, T., What is a Sustainable Healthy Diet?, https://doi.org/http://dx.doi.org/, FCRN Discussion Paper, 1-30 (2014)

Gil, A., Por una Ética del Consumo Política: Florence Kelly y la Liga de Consumidores, Revista de Pensamiento e Investigación Social, 316(14), 311-316 (2008)

Gjerris, M., C. Gamborg y H. Saxe, What to Buy? On the Complexity of Being a Critical Consumer, https://doi.org/10.1007/s10806-015-9591-6, Journal of Agricultural and Environmental Ethics, 29(1), 81-102 (2016)

González, L. y J. David, Consumo y Consumismo Algunos Elementos Traza, Nómadas Revista Crítica de Ciencias Sociales y Jurídicas, 21, 1-19 (2009)

HLPE, Food Losses and Waste in the Context of Sustainable Food Systems. A Report by the High Level Panel of Experts on Food Security and Nutrition of the Committee on World Food Security, https://doi.org/65842315, HLPE REPORT, 1-6 (2014)

INEC. Informe de Resultados sobre la Encuesta de Condiciones de Vida (ECV) 2013 - 2014, 1-28 (2014)

Johnston, J., M. Szabo y A. Rodney, Good Food, Good People: Understanding the Cultural Repertoire of Ethical Eating, https://doi.org/10.1177/1469540511417996, Journal of Consumer Culture, 11(3), 293-318 (2011)

Lara, C., Moral de Mercado Versus Seguridad Alimentaria: Una Aproximación desde La Ética Del Bien Común, https://doi.org/10.4067/S1726-569X2001000200005, Acta Bioethica, 7(2), 233-248 (2001)

Lara, G. y G. Colín, Sociedad de Consumo y Cultura Consumista, Argumentos, México, D.F., (55), $211-216$ (2007)

López, S., L. Marín y S. Ruiz de Maya, Introducing Personal Social Responsibility as a Key Element to Upgrade CSR, https://doi.org/https://doi.org/10.1016/j.sjme.2017.04.001, Spanish Journal of Marketing - ESIC, 18-29 (2017)

Lugo, N., La Salud como Resultado del Consumo, Revista Cubana de Salud Pública, 36(4), 322-329 (2010)

Marquina, P., E. Reficco, Impacto de la Responsabilidad social empresarial en el Comportamiento de Compra y Disposición a Pagar de Consumidores Bogotanos, https://doi.org/10.1016/j.estger.2015.10.001, Estudios Gerenciales, 14(137), 4-23 (2012)

Millán, G., La Ética del Consumo, Consumidor, 76-80 (2012) 
Neira, J., F. Pérez, S. Sanchez y R. Rojas, Study on the Mortality in Ecuador Related to Dietary Factors, https://doi.org/10.3305/nh.2013.28.5.6755, Nutrición Hospitalaria, 28(5), 1732-1740 (2013)

Parada, A., R. Daponte y E. Vázquez, Valoración de la RSC por el Consumidor y Medición de su Efecto sobre las Compras, https://doi.org/10.1590/S0034-759020140105, Revista de Administración de Empresas, 54, 39-52 (2014)

Peattie, K. y S. Peattie, Social Marketing: A pathway to Consumption Reduction? https://doi.org/ 10.1016/j.jbusres.2008.01.033, Journal of Business Research, 62(2), 260-268 (2009)

Regmi, A. y B. Meade, Demand Side Drivers of Global Food Security, https://doi.org/10.1016/j.gfs.2013.08.001, Global Food Security, 2(3), 166-171 (2013)

Rivera, J., E. Bigne y R. Curras, Effects of Corporate Social Responsibility Perception on Consumer Satisfaction with the Brand, https://doi.org/10.1016/j.sjme.2016.06.002, Spanish Journal of Marketing - ESIC, 20(2), 104-114 (2016)

Roper, S. y C. Parker, Doing Well by Doing Good: A quantitative Investigation of the Litter Effect, https://doi.org/10.1016/j.jbusres.2012.02.018, Journal of Business Research, 66(11), 2262-2268 (2013)

Rozin, P., The Meaning of Food in Our Lives: A cross-Cultural Perspective on Eating and Well-Being, https://doi.org/10.1016/S1499-4046(06)60209-1, Journal of Nutrition Education and Behavior, 37 (SUPPL. 2) (2005)

Saad, P., T. Miller y C. Martínez, Impacto de los Cambios Demográficos en las Demandas Sectoriales en América Latina, https://doi.org/10.1590/S0102-30982009000200006, Revista Brasileira de Estudios de Populares, 26 (2), $237-261$ (2009)

Sampaio, E.P., Estudio de las Prácticas Culturales en sus Relaciones con Agricultura, Suelo y Ambiente, https://doi.org/10.1612/inf.tecnol.4003it.08 , Información Tecnológica, 20(3), 113-123 (2009)

Sanchez, L., S.M. Ulloa y M.C. Barragán, Determinación de la Relación entre la Seguridad Alimentaria y la Economía/agricultura Familiar en la Zona Rural del cantón Santo Domingo de los Colorados en Ecuador, Revista Dilemas Contemporaneos: Educación, Política y Valores, ISSN: 2007-7890, 2 (64), 1-18 (2018)

Serrano, M., La Obesidad como Pandemia del Siglo XXI, Fundación MAPFRE (2012)

Serrano, M. y J.C. Serrano, Reporte de Pobreza, https://doi.org/http://www.ecuadorencifras.gob.ec/documentos/webinec/POBREZA/2016/Marzo_2016/Info, Inec, 1-9 (2016)

Smyth, S., P. Phillips y W. Kerr, Food Security and the Evaluation of Risk, https://doi.org/10.1016/j.gfs.2014.08.001, Global Food Security, 4, 16-23 (2015)

Sotorno, A., Hacia una Sociedad de Consumo Etico, Gestión, 150(March), 29-33 (2007)

Spangenberg, J., Institutional Change for Strong Sustainable Consumption: Sustainable Consumption and the Degrowth Economy, https://doi.org/10.1080/15487733.2014.11908125, Sustainability: Science, Practice, and Policy, 10(1), 62-77 (2014)

Sudbury, L. y F. Kohlbacher, Ethically Minded Consumer Behavior: Scale Review, Development, and Validation, https://doi.org/10.1016/j.jbusres.2015.11.005, Journal of Business Research, 69(8), 2697-2710 (2016)

Vermeir, I. y W. Verbeke, Sustainable Food Consumption Among Young Adults in Belgium: Theory of Planned Behaviour and the Role of Confidence and Values, https://doi.org/10.1016/j.ecolecon.2007.03.007, Ecological Economics, 64(3), 542-553 (2008) 
